\title{
LES JUGES ET LES ÉLECTIONS DANS L'ÉGYPTE POST MOUBARAK : ACTEURS OU VICTIMES DU POLITIQUE?
}

\author{
Nathalie Bernard-Maugiron \\ L'Harmattan | Confluences Méditerranée
}

\author{
2012/3 - N82 \\ pages 117 à 132
}

ISSN 1148-2664

Article disponible en ligne à l'adresse:

http://www.cairn.info/revue-confluences-mediterranee-2012-3-page-117.htm

Pour citer cet article :

Bernard-Maugiron Nathalie, «Les juges et les élections dans l'Égypte post Moubarak : acteurs ou victimes du politique? »,

Confluences Méditerranée, 2012/3 №82, p. 117-132. DOI : 10.3917/come.082.0117

Distribution électronique Cairn.info pour L'Harmattan.

(c) L'Harmattan. Tous droits réservés pour tous pays.

La reproduction ou représentation de cet article, notamment par photocopie, n'est autorisée que dans les limites des conditions générales d'utilisation du site ou, le cas échéant, des conditions générales de la licence souscrite par votre établissement. Toute autre reproduction ou représentation, en tout ou partie, sous quelque forme et de quelque manière que ce soit, est interdite sauf accord préalable et écrit de l'éditeur, en dehors des cas prévus par la législation en vigueur en France. II est précisé que son stockage dans une base de données est également interdit. 


\section{Nathalie Bernard-Maugiron}

Directrice de recherche. IRD, UMR 201, Développement et Sociétés (Université Paris $1 /$ IEDES et IRD).

\section{Les juges et les élections dans l'Egypte post Moubarak : acteurs ou victimes du politique?}

Les décisions des juridictions égyptiennes relatives aux processus électoraux de l'Égypte post-Moubarak ont été perçues par les partisans des Frères musulmans comme hostiles au nouveau pouvoir en place et ont entraîné des accusations de collusion des magistrats avec l'armée et les anciens du régime de Moubarak. Cet article s'attache à rappeler, d'une part, que cette instrumentalisation du politique n'est pas nouvelle et qu'elle s'était exercée contre le régime de Moubarak, et à montrer, d'autre part, que les décisions controversées de la cour constitutionnelle s'inscrivent dans la droite ligne de sa jurisprudence. Il s'interroge sur le statut d'acteur ou de victime des magistrats dans ce processus de politisation du judiciaire.

T e 14 juin 2012, deux jours avant le premier tour des élections présidentielles, la Haute Cour constitutionnelle égyptienne invalida la loi organisant les élections législatives, pour rupture du principe d'égalité entre candidats de partis politiques et indépendants. Le lendemain, le maréchal Tantawi, chef du Conseil suprême des forces armées, à la tête du pays depuis la chute de Hosni Moubarak, dissout l'Assemblée du peuple par 
décret. Trois semaines plus tard, le 8 juillet 2012, le président Mohammed Morsi, qui venait de prendre ses fonctions ${ }^{1}$, abrogea le décret de Tantawi et réinstitua dans ses fonctions la chambre basse du Parlement en lui demandant de reprendre ses travaux. La Haute Cour constitutionnelle s'empressa de se réunir et déclara que ce décret présidentiel devait être privé d'effets car la composition de l'Assemblée du peuple était nulle depuis le jour des élections. La chambre tint une ultime (?) réunion d'un quart d'heure, à l'issue de laquelle son président décida de saisir la Cour de cassation du différend relatif aux effets de la décision du juge constitutionnel. Quelques jours plus tard, la Cour de cassation se déclara incompétente pour connaitre de cette affaire.

Ces différentes décisions de justice suscitèrent de vives critiques et réactions de rejet de la part des partisans des Frères musulmans, des duels musclés opposèrent des professeurs de droit constitutionnel et des débats passionnés agitèrent les médias. On vit même des manifestants protester devant le siège des hautes juridictions et accuser les juges d'être aux ordres des militaires et des anciens du régime. Cet imbroglio juridico-judiciaire reflète de façon certes caricaturale mais relativement exemplaire le rôle que les juridictions égyptiennes ont été amenées à jouer depuis la chute de Moubarak tout au long du processus de transition. Elles ont non seulement dû juger les hauts responsables de l'ancien régime - au premier plan desquels l'ex-président lui-même -, tenter d'obtenir la récupération des fonds indûment détournés par les anciens hauts responsables corrompus, juger les policiers soupçonnés d'avoir tiré sur des manifestants ainsi que les jeunes révolutionnaires accusés d'avoir attaqué des policiers, mais également superviser les processus électoraux et se prononcer sur des questions encore plus directement politiques, comme la validité de la composition de l'Assemblée constituante, la possibilité ou non pour un ancien premier ministre de Moubarak de se présenter aux élections présidentielles ou, comme on l'a vu, invalider l'assemblée législative. Cette implication des tribunaux dans la gestion du politique n'est toutefois pas nouvelle. Sous Moubarak, déjà, les magistrats avaient été accusés de sortir de leur mandat pour s'ingérer dans le politique. Mais la politisation du judiciaire s'est encore accentuée après la chute du président et la gestion chaotique de la période de transition par les généraux. Les tribunaux se sont ainsi trouvés instrumentalisés dans la lutte pour le pouvoir qui oppose les Frères musulmans au Conseil suprême des 
Les juges et les élections dans l'Égypte post Moubarak : acteurs ou victimes du politique?

forces armées, dans laquelle on peut se demander s'ils n'ont pas été davantage victimes que parties.

\section{Les juges et la supervision des élections avant la chute de Moubarak}

Il est quelque peu paradoxal que les magistrats égyptiens soient aujourd'hui accusés d'être des soutiens de l'ancien régime et de l'armée, alors que sous Moubarak ils étaient au contraire considérés comme l'un des seuls véritables contre-pouvoirs à l'autoritarisme du président ${ }^{2}$. De même, ils sont aujourd'hui accusés d'être hostiles aux islamistes, alors que le régime de Moubarak avait tenté de discréditer le mouvement des juges réformistes en affirmant qu'ils étaient infiltrés et manipulés par les Frères musulmans ${ }^{3}$.

Les juges égyptiens s'étaient ainsi mobilisés en 2005/2006 pour des élections transparentes et une réelle indépendance de la magistrature, allant jusqu'à recourir à la menace du boycott de la supervision des élections pour tenter de faire fléchir le pouvoir. Suite à une décision de la Haute Cour constitutionnelle de 2000, les juges supervisaient en effet le déroulement des élections à l'intérieur des bureaux de vote, ce qui avait fait d'eux les témoins directs et impuissants d'une multitude de pratiques frauduleuses, que certains d'entre eux n'avaient pas hésité à dénoncer. Refusant d'être instrumentalisés pour cautionner des élections truquées, ils avaient alors exigé des garanties de transparence du processus électoral et la supervision intégrale du processus électoral, depuis l'élaboration des listes d'électeurs jusqu'à la proclamation des résultats. En 2006, devant la résistance du régime, ils avaient été jusqu'à recourir à l'organisation d'un sit-in au Caire devant leur club ${ }^{4}$. Ce dernier devint d'ailleurs un lieu de mobilisation politique et d'expression par les citoyens de leur soutien aux juges. Malgré cette forte mobilisation, les juges n'avaient pas obtenu satisfaction. En 2007, la supervision des élections leur avait même été retirée par un amendement constitutionnel, qui n'exigea plus la présence de juges que pour le seul décompte des voix. Toute une série de mesures de rétorsions furent également prises à l'encontre du Club des juges, principal vecteur du soulèvement des magistrats, tant et si bien qu'en 2009 le président et le conseil d'administration sortants du club perdirent 
les élections et qu'une majorité conservatrice, réputée proche du régime, reprit en mains la direction. Si la supervision judiciaire avait permis aux juges de mesurer leur force sur la scène politique et de rallier autour d'eux l'ensemble des forces de l'opposition et toute la société civile, elle avait aussi constitué un premier pas vers une entrée en politique du judiciaire.

La deuxième revendication principale du mouvement de protestation des juges de 2005 était de renforcer l'indépendance de la magistrature. Selon les juges, la loi sur le pouvoir judiciaire donnait les moyens au pouvoir exécutif d'intervenir dans les affaires de la justice et devait être amendée. Ils demandaient notamment que la composition du Conseil suprême de la magistrature soit modifiée et que la moitié au moins de ses membres soit élue par leurs pairs au lieu de siéger ex officio sur la base des fonctions qu'ils occupaient. Ils proposaient également que le président de la Cour de cassation soit choisi au scrutin secret par l'Assemblée générale de la Cour, au lieu d'être nommé par décret présidentiel. Les assemblées générales des tribunaux devaient se voir retirer la possibilité de déléguer au président de leur juridiction une partie de leurs pouvoirs, en particulier la répartition des affaires entre les différentes chambres ${ }^{5}$.La direction de l'inspection judiciaire devait être rattachée au Conseil suprême de la magistrature et non plus au ministère de la Justice ; les conditions de mise à disposition et de détachement des juges devaient être réformées : un des moyens de sanctionner un juge indocile était ainsi de le mandater pour accomplir des tâches ne relevant pas des fonctions judiciaires ${ }^{6}$. Inversement, certains détachements auprès de ministères ou d'administrations publiques, où les salaires étaient très élevés, ou encore auprès de gouvernements étrangers, en particulier dans les pays du Golfe, pouvaient servir à remercier un juge particulièrement loyal. Certains auraient aussi souhaité que le procureur général soit nommé par le Conseil suprême de la magistrature et non plus par le président de la République. La loi sur le pouvoir judiciaire fut certes amendée en 2006, mais les juges réformistes n'obtinrent que des concessions très partielles, très loin de leurs attentes.

Les juges de la cour constitutionnelle, de leur côté, n'avaient pas participé à la fronde de 2005, n'étant pas sollicités pour superviser les scrutins électoraux et leur loi d'organisation leur donnant de solides garanties d'indépendance par rapport au ministère de la Justice. Ils sont nommés selon un mécanisme de cooptation : le 
président de la République doit choisir entre deux candidats, dont l'un est proposé par l'assemblée générale de la Cour et l'autre (souvent le même) par son président. Le Conseil suprême des organes judiciaires, composé des présidents de tous les corps judiciaires ainsi que du ministre de la Justice et présidé par le président de la République, donne un avis facultatif. Une fois nommés, les juges sont inamovibles. Tous les juges de la Cour, sauf un ${ }^{7}$, ont été choisis au sein des autres tribunaux, particulièrement la Cour de cassation et le Conseil d'État. La Cour a son propre budget et les salaires perçus par ses membres sont bien supérieurs à ceux versés aux juges des juridictions ordinaires. Il est vrai toutefois que le président de la cour constitutionnelle est nommé par le président de la République à sa totale discrétion, l'avis du Conseil suprême des organes judiciaires n'étant même pas exigé. Cela permet au chef de l'État de choisir des personnes en lesquelles il a toute confiance. Pendant très longtemps, la tradition en vigueur au sein de la Cour a été de nommer à sa tête son membre le plus ancien. Mais cette tradition s'est perdue au début des années 2000, lorsque Moubarak a commencé à choisir des présidents au sein d'autres juridictions. En août 2001, ainsi, fut nommé à sa tête le président de la Cour de cassation, qui avait longtemps dirigé le département législatif au sein du ministère de la Justice ${ }^{8}$.

\section{La supervision judiciaire des processus électoraux après la chute de Moubarak}

La Déclaration constitutionnelle du 30 mars 2011, adoptée 6 semaines après la chute de Moubarak, réinstitua la supervision judiciaire des élections supprimée en 2007. C'est également à des juges que fut confiée l'organisation du processus électoral au sein des commissions électorales. Les élections législatives furent ainsi supervisées par une Haute commission électorale, composée de magistrats et ayant à sa tête le président de la Cour d'appel du Caire, tandis que la commission des élections présidentielles, entièrement composée de juges, était présidée par le président de la Haute Cour constitutionnelle. Grâce à une décision du Conseil d'État d'octobre 2011, les Égyptiens de l'étranger purent voter pour la première fois, à la fois aux législatives et aux présidentielles. Si des irrégularités 
furent constatées par les ONG locales et internationales qui supervisèrent les processus électoraux, on fut quand même loin des phénomènes de fraudes massives qu'avait connus le pays lors des élections législatives de 2010.

Le 21 février 2012, la Haute Cour constitutionnelle fut saisie par le Conseil d'État de la constitutionnalité de la loi électorale pour les législatives. Cette dernière réservait $2 / 3$ des sièges aux partis politiques sur la base du scrutin de liste, alors que les candidats indépendants, non affiliés à un parti politique reconnu, ne pouvaient concourir au scrutin uninominal que sur le tiers restant, sur lequel les candidats de partis politiques pouvaient également se présenter ${ }^{9}$. Dans sa décision du 14 juin 2012, la Cour estima qu'il y avait rupture du principe d'égalité des chances entre candidats, les membres des partis politiques ayant la possibilité de remporter des sièges suivant le scrutin de listes ou suivant le scrutin individuel, alors que les indépendants ne pouvaient se présenter que sur les sièges individuels, sur lesquels ils se retrouvaient même en compétition avec les candidats des partis politiques. Ces derniers bénéficiaient, en plus, du soutien financier et moral du parti auquel ils appartenaient, ce qui n'était pas le cas des candidats indépendants. Cette décision vint confirmer la jurisprudence antérieure de la Cour : en 1987 et 1990, la cour constitutionnelle avait en effet déjà déclaré inconstitutionnelles des lois électorales analogues, pour des motifs similaires ${ }^{10}$.

$\mathrm{Si}$ le dispositif de la décision se limita à invalider certaines dispositions de la loi électorale, le juge constitutionnel précisa toutefois dans les motifs de son jugement que puisque les élections législatives s'étaient tenues sur la base d'une loi inconstitutionnelle, il en découlait nécessairement que la composition de la totalité de l'Assemblée devait être considérée comme nulle dès le jour des élections, ce qui entraînait sa dissolution de plein droit et sans qu'il soit nécessaire d'adopter une mesure spécifique. En $1987^{11}$ et $1990^{12}$, Moubarak avait lui-même dissout l'Assemblée du peuple suite aux décisions de la Haute Cour constitutionnelle. Malgré la clarté de la décision de la cour constitutionnelle, la classe politique se divisa sur les conséquences à en tirer : est-ce que seul le tiers du parlement, élu sur la base du scrutin individuel, devait être dissout ? Est-ce que la dissolution devait être prononcée immédiatement ? Est-ce que les $2 / 3$ restants pouvaient continuer à siéger en attendant le résultat des éventuelles élections partielles ? Est-ce qu'en cas de dissolution, il fallait organiser un référendum, comme le prévoyait la constitu- 
tion de 1971 ? Bien que la Déclaration constitutionnelle de mars 2011 n'ait pas prévu de procédure de dissolution du parlement, les Frères musulmans affirmaient qu'il était nécessaire d'organiser un référendum car l'Assemblée du peuple avait été élue par le peuple.

Le Conseil suprême des forces armées décida de dissoudre la totalité de l'Assemblée du peuple, sans organiser de référendum. Suite à l'abrogation par le président Morsi de ce décret le 9 juillet, l'assemblée générale de la Haute Cour constitutionnelle se réunit dans l'urgence, sur la base de sa loi d'organisation qui lui donne compétence pour trancher les litiges nés de l'exécution de ses propres décisions. La Cour rappela qu'elle avait affirmé dans sa décision du 14 juin que la totalité de l'Assemblée du peuple était nulle depuis le jour des élections. En conséquence, la décision du président Morsi constituait un obstacle matériel à la mise en oeuvre de son jugement et il fallait en stopper l'exécution. Parallèlement, des recours furent déposés devant le Conseil d'État contre le décret du président Morsi. L'Assemblée du peuple, quant à elle, se réunit le 10 juillet 2012 pour un quart d'heure, le temps pour son président de renvoyer tout le dossier à la Cour de cassation afin qu'elle examine la validité du mandat des députés, conformément à l'article 40 de la Déclaration constitutionnelle de mars 2011. Mais la Cour de cassation se déclara incompétente pour connaître de l'affaire. Le Conseil d'État, quant à lui, confirma le 17 juillet 2012 que le décret de Mohammed Morsi annulant la dissolution de l'Assemblée du peuple constituait un «obstacle matériel » à la mise en œuvre de la décision de la Haute Cour constitutionnelle et relevait de la compétence de cette dernière.

La Haute Cour constitutionnelle eut également à se prononcer sur le processus électoral dans le cadre des présidentielles. Après l'annonce le 6 avril 2012 de la candidature surprise de Omar Soliman, ancien vice-président et ministre de la Défense de Moubarak, et de crainte de le voir remporter les élections, le Parlement avait adopté dans l'urgence le 12 avril 2012 une loi amendant la loi de 1956 sur l'exercice des droits politiques, afin de priver toute personne ayant occupé les fonctions de président, vice-président, premier ministre ou de direction du PND dans les 10 années antérieures au 11 février 2011, de l'exercice de ses droits politiques pendant 10 ans.

La Commission des élections présidentielles appliqua tout d'abord la loi à Ahmed Chafiq, dernier premier ministre de Moubarak, et le 24 avril 2012 jugea sa candidature irrecevable ${ }^{13}$. Ce dernier fit 
appel de cette décision devant la même commission électorale. La Commission décida de soumettre les amendements à la Haute Cour constitutionnelle pour contrôle de leur conformité à la constitution ${ }^{14}$ et, dans l'attente, autorisa finalement Ahmed Chafik à se présenter aux élections ${ }^{15}$.

C'est entre les deux tours de la présidentielle, et alors que Ahmed Chafik était en ballotage avec le candidat des Frères musulmans, que la Haute Cour constitutionnelle rendit sa décision. Elle déclara la loi sur l'exclusion des droits politiques inconstitutionnelle pour plusieurs motifs : elle infligeait une peine de façon rétroactive, établissait une présomption légale de culpabilité alors que les peines doivent résulter d'une condamnation judiciaire, infligeait une peine sur la base des fonctions occupées et non en raison d'actions commises et, enfin, violait le principe d'égalité devant la loi en stigmatisant certaines fonctions de façon arbitraire. Cette décision était conforme à la jurisprudence de la Cour dans les années 80 , lorsqu'elle avait déclaré inconstitutionnelles des dispositions similaires de la loi de 1978 « sur la protection du front intérieur et de la paix sociale », qui prévoyaient la déchéance des droits politiques, d'une part de tous ceux qui avaient participé à la direction d'un des partis politiques au pouvoir avant la Révolution de 1952 ou qui avait occupé des fonctions ministérielles ${ }^{16}$, puis une autre disposition de la même loi qui visait cette fois-ci des dirigeants nassériens qu'elle privait également de leurs droits politiques ${ }^{17}$. La décision de juin 2012 fut toutefois attaquée très violemment par les adversaires de Chafik, au premier rang desquels les Frères musulmans et les salafistes, qui accusèrent la Cour d'avoir pris parti pour le candidat de l'armée.

Enfin, les juridictions administratives eurent elles-aussi à se prononcer sur le processus électoral, touchant cette fois-ci à l'élection des membres de l'assemblée constituante. La Déclaration constitutionnelle de mars 2011 prévoyait que les membres élus des deux chambres du parlement se réuniraient pour choisir les 100 membres de la constituante. Le texte ne fixant pas les critères de nomination, le Conseil suprême des forces armées et les Frères musulmans avaient mené un bras de fer pendant des mois pour en contrôler la composition. Le 17 mars 2012, les deux chambres du parlement, à majorité islamiste, se réunirent et décidèrent de choisir la moitié des membres de la constituante au sein du parlement. Les représentants de la plupart des partis libéraux et de gauche refusèrent de participer au vote, estimant que la constituante devait refléter 
Les juges et les élections dans l'Égypte post Moubarak : acteurs ou victimes du politique?

la diversité des forces politiques, sociales et communautaires de la population et non être dominée par une seule force politique. Une coalition de juristes et partis politiques libéraux déposa un recours devant le Conseil d'État pour inconstitutionnalité de la composition de la constituante. Le 10 avril 2012, la Haute Cour administrative du Conseil d'État donna raison aux requérants, estimant que l'article 60 de la Déclaration constitutionnelle du 30 mars 2011 avait donné mandat au parlement d'« élire » les membres de la constituante, sans préciser qu'une fraction devait être choisie en son sein. Or, si le constituant avait voulu qu'une partie des membres de la constituante soit choisie au sein des parlementaires, il l'aurait clairement spécifié dans la disposition, ajouta le Conseil d'État. Par ce raisonnement a contrario quelque peu audacieux, le juge administratif invalida donc la composition de la constituante. Il est intéressant également de souligner que le juge administratif déclara le recours recevable, estimant que la nomination par le parlement des membres de la constituante devait être considérée comme une décision administrative, soumise au contrôle du Conseil d'État, et non comme un acte parlementaire, exclu de tout recours juridictionnel. Cette solution juridique à l'impasse politique infligea un revers aux partis islamistes.

Il est vrai également que les déclarations de certains membres de la magistrature purent renforcer le sentiment de manque d'indépendance du judiciaire, comme celles du président du Club des juges, se dressant début juin 2012 contre l'Assemblée du peuple qui avait osé critiquer le jugement de la Cour criminelle du Caire dans le procès Moubarak, ou fixant un ultimatum le 9 juillet 2012 au président Morsi pour qu'il retire son décret invalidant la dissolution de l'Assemblée du peuple. Ces déclarations sont d'autant plus étonnantes que ce même président avait été élu en 2009 à la tête du Club des juges sur un programme condamnant l'utilisation des médias par les juges pour faire des déclarations politiques. Ces prises de position furent condamnées par un grand nombre de magistrats, qui prirent clairement leur distance avec leur auteur, affirmant qu'elles n'étaient en aucun cas représentatives du pouvoir judiciaire. Les propos de la seule femme juge à la Cour constitutionnelle, déclarant lors d'une interview à un journal américain qu'elle avait conseillé aux généraux de ne pas remettre le pouvoir aux civils avant qu'une nouvelle constitution ne soit élaborée ${ }^{18}$, contribuèrent également à écorner l'image de la magistrature. 
Les juridictions pénales qui ne donnent pas suite aux plaintes déposées contre des officiers et des policiers furent aussi fortement critiquées et accusées de complaisance envers le pouvoir ${ }^{19}$. Les tribunaux furent également l'objet de toutes les suspicions, lorsque le président de la Cour d'appel du Caire intervint personnellement pour obtenir la remise en liberté d'étrangers mis en examen dans l'affaire du financement des $\mathrm{ONG}$, et que l'assemblée générale de la Cour d'appel lui renouvela malgré tout sa confiance.

\section{Les juges : acteurs ou victimes du politique?}

$\mathrm{Si}$ les décisions de la cour constitutionnelle, tant en ce qui concerne la constitutionnalité de la loi électorale que la loi d'exclusion des droits politiques, étaient conformes à sa jurisprudence antérieure, il n'en reste pas moins que la célérité avec laquelle la Cour a examiné les deux requêtes et le choix stratégique du moment où elles ont été rendues, ont pu étonner les observateurs. Saisie de la loi électorale en février 2012 et de la loi d'exclusion politique en avril 2012, la Cour a rendu ses décisions dans les deux cas le 14 juin 2012, soit 4 mois après sa saisine dans un cas et 2 mois seulement dans l'autre, ce qui constitue un délai exceptionnellement rapide. Certes, dans les 2 cas, la décision de la Cour n'a fait que conforter une jurisprudence antérieure, mais il n'en reste pas moins que ces délais sont très courts. La loi d'organisation de la Cour ne lui fixe pas de délai pour rendre ses décisions et il n'est pas rare que la Cour mette des années à se prononcer. Cette prise en considération du contexte politique le plus propice pour rendre ses décisions n'est toutefois pas nouvelle. Sous Moubarak, ainsi, la Cour gardait souvent dans ses tiroirs pendant des années des décisions que l'on disait prêtes à être rendues publiques, dans l'attente du contexte politique le plus opportun. Ce moment pouvait d'ailleurs ne jamais se présenter, comme ce fut le cas pour la loi sur la nationalité qui interdisait aux mères égyptiennes de transmettre leur nationalité à leurs enfants, qui finit par être amendée en 2004 sans que la Haute Cour constitutionnelle n'ait jamais rendu sa décision, ou la loi sur l'état d'urgence. La décision de la Cour de 2000 sur la supervision judiciaire des élections n'avait-elle pas été rendue 10 ans après que le requérant l'ait saisie de la constitutionnalité de la loi électorale qui avait organisé les élections législatives de 1990, permettant ainsi 
Les juges et les élections dans l'Égypte post Moubarak : acteurs ou victimes du politique?

à deux législatures de terminer leurs travaux, avant que la Cour estime que le moment était venu de rendre sa décision ?

Il est vrai que la plupart des décisions prises par la Haute Cour constitutionnelle et le Conseil d'État vont à l'encontre des intérêts des Frères musulmans. Mais ces jugements doivent-ils nécessairement s'expliquer, comme l'ont fait certains acteurs ou médias, par le fait que les juges sont aux ordres du Conseil suprême des forces armées? Tout juge est influencé par ses propres convictions et sa conception de la justice. Dans le cas des magistrats égyptiens, cette réticence sinon hostilité à l'égard des Frères musulmans et des salafistes peut s'expliquer par différents facteurs. Tout d'abord, tous les magistrats actuellement en poste dans les tribunaux sont entrés dans la magistrature sous l'ancien régime. Or, les conditions de recrutement des magistrats, tant dans les juridictions ordinaires qu'au Conseil d'État, sont telles que tous les jeunes recrutés proviennent d'un même milieu social, la moyenne bourgeoisie. La loi exige qu'ils présentent toutes les garanties de moralité et de bonne réputation, ce qui se traduit en pratique par un contrôle sévère opéré à l'entrée dans la magistrature, avec en particulier une enquête des services de sécurité menée sur chaque candidat et la mise à l'écart systématique de ceux qui seraient issus de familles des couches basses de la population, qui auraient des proches dans les milieux islamistes, gauchistes ou qui auraient fait l'objet d'une condamnation pénale. Cet écrémage fait que les juges sont souvent recrutés de père en fils, dans des milieux sociaux conservateurs mais plutôt libéraux au niveau politique, avec un sens profond de l'importance de leur statut de magistrat et des valeurs de l'État de droit et de la souveraineté de la loi.

Beaucoup sont donc effectivement plutôt proches par leurs convictions politiques, sociales et religieuses des hommes de l'ancien régime et de l'armée. Ils partagent le conservatisme des hommes de Moubarak et leur crainte de voir arriver les islamistes au pouvoir. Très souvent musulmans pratiquants, ils n'ont cependant pour la plupart aucune envie de se retrouver dans un État théocratique, si tant est que les Frères musulmans veuillent vraiment instituer un tel État, ni de devoir appliquer des lois directement inspirées de la shari'a, comme un code pénal prévoyant des châtiments corporels. Ces juges formés dans les facultés de droit des Universités égyptiennes, n'ont pour la plupart qu'une connaissance très sommaire du droit musulman. La jurisprudence de la cour constitutionnelle 
relative à l'interprétation de l'article 2 de la Constitution de 1971 ("Les principes de la shari'a islamique sont la source principale de la législation ») montre d'ailleurs comment cette juridiction a recouru à divers mécanismes juridiques pour limiter la portée de cette disposition et, partant, la place de la shari'a dans le système juridique égyptien. La perspective d'un partage du pouvoir judiciaire avec des hommes de religion ne doit pas non plus les motiver particulièrement.

Il est vrai également que pendant les 3 mois où elle s'est réunie, l'Assemblée du peuple dominée par les islamistes n'a pas adopté un comportement propre à apaiser les craintes des magistrats. Tout d'abord, divers projets de réorganisation de la Haute Cour constitutionnelle ont été déposés par des députés salafistes, qui visaient à réduire ses attributions ou à priver cette juridiction de toute sa nature. L'un des projets prévoyait ainsi que les décisions de la Haute Cour constitutionnelle devraient être ratifiées par le Parlement pour avoir force obligatoire. En ce qui concerne les tribunaux ordinaires, l'assemblée n'a pas examiné ni à fortiori adopté les projets de réforme de la loi sur le pouvoir judiciaire qui visaient à renforcer l'indépendance des magistrats par rapport au pouvoir exécutif. Il est alors un peu paradoxal que les Frères musulmans accusent les juges de ne pas être indépendants, alors même qu'ils n'ont pas saisi l'occasion qui leur était donnée de renforcer cette indépendance.

Il ne faut pas oublier non plus que les juges ne se sont pas saisis de ces affaires de leur propre initiative, ce sont les acteurs politiques eux-mêmes, incapables de résoudre leurs différends par le dialogue et le consensus, qui se sont adressés à la justice pour qu'elle tranche leurs conflits. Cette instrumentalisation politique des tribunaux n'est d'ailleurs pas nouvelle. Sous Moubarak, déjà, les tribunaux étaient régulièrement sollicités par diverses catégories d'acteurs (partis d'opposition, ONG, groupes islamistes), dont les actions en justice étaient sous-tendues par des logiques plus politiques que juridiques : privés de lieux d'expression et de contestation, les opposants avaient pris l'habitude de défendre leurs opinions devant les tribunaux. Ils avaient ainsi pu obtenir des réformes qu'ils n'auraient pu atteindre par le biais d'amendements législatifs, le parlement étant entièrement aux mains du parti du président. Le régime avait d'ailleurs tenté de lutter contre ce phénomène, refusant d'exécuter certaines décisions de justice ou soustrayant certaines affaires à la compétence des tribunaux ordinaires pour les confier à des juridictions d'exception. 
Après la chute de Moubarak, les juridictions égyptiennes ont certes pris des décisions qui allaient à l'encontre des intérêts des Frères musulmans et qui ont été interprétées comme favorables au Conseil suprême des forces armées, mais elles se sont également prononcées contre les intérêts de l'ancien régime. Il ne faut pas oublier ainsi que c'est une décision du Conseil d'État qui a dissout le PND en avril 2011, et que les conseils municipaux, dominés par les hommes du PND, ont également été dissous par une décision du juge administratif en juin 2011. La cinquantaine de nouveaux partis politiques, y compris le parti Justice et Liberté ainsi que le parti salafiste al-Nour ont été reconnus par une décision du comité des partis politiques dans sa nouvelle composition, entièrement formée de membres du judiciaire. De même, c'est le Conseil d'État qui a déclaré illégaux un certain nombre de décrets de privatisation du secteur public pris par Moubarak. Le fait que l'armée a eu plus que jamais recours aux tribunaux militaires après le 11 février 2011 pour juger les jeunes manifestants montre également que les généraux n'avaient pas une confiance absolue dans le soutien inconditionnel des magistrats.

\section{Conclusion}

Avec la nomination d'un ancien juge réformiste à la vice-présidence de la République et de son frère au poste de ministre de la Justice, l'intervention des juges dans le politique est amenée à prendre de nouvelles formes. Ces nominations ont en effet été perçues comme des tentatives par les Frères musulmans de se rallier une partie de la magistrature égyptienne, même si l'une des premières mesures adoptées par le nouveau ministre a été de renforcer l'indépendance de la magistrature en rattachant l'inspection judiciaire au Conseil suprême de la magistrature, une des plus anciennes revendications des juges réformistes

La Cour constitutionnelle va se heurter à la précarité de son assise normative. Elle n'est en effet pas à l'abri de l'adoption de mesures de rétorsion de la part des partis islamistes, que ce soit sous la forme d'une amputation de ses attributions ou d'une «fournée de juges » islamistes, qui viendraient modifier l'équilibre au sein de la Cour ${ }^{20}$. L'avant-projet du nouvel article 2 de la constitution élaboré par l'Assemblée constituante à majorité islamiste prévoit ainsi qu'al- 
Azhar devra avoir le dernier mot en ce qui concerne l'interprétation de la shari'a. Cet article, proposé par les salafistes, vise à retirer à la Cour constitutionnelle son pouvoir d'interprétation de l'article 2. Dès son arrivée au pouvoir, le nouveau ministre de la Justice a également tenu des propos hostiles à la Cour, et lancé l'idée de réunifier le système juridictionnel en réintégrant le juge constitutionnel à l'ordre judiciaire ordinaire. Le président Morsi a également aboli en août 2012 la déclaration constitutionnelle additionnelle adoptée par le Conseil suprême des forces armées en juin, qui donnait à la Haute Cour constitutionnelle des pouvoirs exorbitants tout au long du processus d'élaboration de la constitution.

Louis Favoreu, grand constitutionnaliste français, faisait remarquer que " le juge constitutionnel doit savoir jusqu'où ne pas aller trop loin. Il faut qu'il adopte des techniques de contrôle progressivement acceptées. Le pouvoir du juge constitutionnel ne peut véritablement s'exercer que s'il se sent soutenu par l'opinion et que si l'équilibre qu'il établit entre les pouvoirs est accepté ${ }^{21}$. De même, Marc Verdussen, autre grand constitutionnaliste, faisait remarquer que les décisions d'un juge constitutionnel « seront d'autant plus acceptables qu'elles procèdent d'une attitude de prudence constructive $^{22}$ ». La Haute Cour constitutionnelle, et les autres juridictions égyptiennes, ont été entrainées hors de leurs limites. Elles sont les victimes collatérales de la gestion fébrile et peu cohérente de la période de transition par le Conseil suprême des forces armées. Quel sera l'effet à long terme que toutes ces polémiques auront sur le capital de confiance et de respect dont bénéficiaient les juges au sein de la population égyptienne ? La dégradation de leur image, sérieusement écornée par toutes ces affaires, aura-t-elle des effets à long terme ? Seront-ils toujours considérés comme les gardiens des droits et libertés et de la transparence des élections ? Sinon, qui pourra les remplacer?

1. En l'absence d'Assemblée du peuple, dissoute, c'est devant la Haute Cour constitutionnelle que le président Morsi prêta serment, deux semaines après les décisions de cette dernière relatives à la loi électorale et à la loi sur l'exercice des droits politiques. Pour marquer son hostilité envers le juge constitutionnel, il se rendit d'abord le 29 juin 2012 à la Place Tahrir pour prêter serment devant le peuple, avant de prêter serment le lendemain devant la Haute Cour constitutionnelle. 
Les juges et les élections dans l'Égypte post Moubarak : acteurs ou victimes du politique?

2. Des conflits avaient déjà opposé les magistrats au pouvoir exécutif dans le passé, en particulier sous Nasser, et s'étaient soldés par la dissolution en 1969 du conseil d'administration du Club des juges et la mise à la retraite ou la mutation à des fonctions non judiciaires de près de 200 magistrats.

3. Ces accusations reposaient notamment sur le fait que la plupart des fraudes dénoncées par les juges avaient été commises en faveur des candidats du parti national démocratique (PND) au pouvoir, au détriment des candidats associés aux Frères musulmans. Elles étaient également dues à l'hostilité manifestée par l'ensemble du corps de la magistrature à l'ouverture de la profession aux femmes.

4. À l'origine simple organisation de défense des intérêts corporatistes et matériels des juges des tribunaux civils, le Club des juges est vite devenu une association professionnelle non officielle.

5. Les juges réformistes affirmaient que cette pratique rendait possible l'attribution d'une affaire spécifique à un juge déterminé, alors que la distribution des affaires entre chambres devrait se faire selon des règles abstraites et générales.

6. Ces détachements procèdent d'une décision du président de la République, après avis de l'assemblée générale du tribunal auquel appartient le magistrat ou du procureur général s'il s'agit d'un membre du parquet, et l'accord du Conseil suprême de la magistrature.

7. Le seul membre de la Cour qui n'ait pas été choisi au sein de la magistrature est Tahani al-Gibali, la seule femme juge de la Cour constitutionnelle, nommée en 2003 alors qu'elle était avocate.

8. En 2006, le président de la Haute Cour constitutionnelle, qui venait d'atteindre l'âge de la retraite après 3 années passées à la tête de cette juridiction, fut nommé ministre de la Justice. Il fut remplacé par l'ancien procureur général. De 2009 à juin 2012, la Cour fut présidée un ancien membre du parquet militaire et jusque là président du Tribunal de grande instance du sud du Caire.

9. À l'origine, le projet de loi prévoyait une répartition à égalité des sièges entre candidats des partis politiques et indépendants. Il était ensuite passé en septembre 2011 à $2 / 3$ (partis politiques) et $1 / 3$ (indépendants). Devant les craintes de voir les anciens membres du PND remporter de façon massive les sièges des indépendants, le Conseil suprême des forces armées avait finalement choisi, en octobre 2011, d'autoriser les partis politiques à se présenter sur les sièges des indépendants.

10. Décisions du 16 mai 1987 et du 19 mai 1990. Pour éviter la déclaration d'inconstitutionnalité, le Conseil suprême des forces armées avait pourtant pris soin d'amender la Déclaration constitutionnelle le 25 septembre 2011, pour y faire figurer la répartition $2 / 3$ (partis politiques) et $1 / 3$ (indépendants), sans préciser toutefois que les partis politiques pouvaient concourir sur les sièges des indépendants.

11. Le président avait même anticipé la décision de la Haute Cour constitutionnelle et dissout l'Assemblée du peuple dès février 1987, alors que la décision de la cour constitutionnelle ne fut rendue que le 16 mai 1987 et qu'une nouvelle assemblée avait déjà été élue le 6 avril 1987.

12. Moubarak n'avait toutefois pas dissout immédiatement l'Assemblée du peuple, puisque la décision de la Haute Cour constitutionnelle avait été rendue le 19 mai 1990 alors que le référendum n'avait été organisé que le 11 octobre 1990, ce qui avait permis à l'assemblée de terminer son mandat.

13. La candidature de Omar Soliman fut finalement disqualifiée sur d'autres 
fondements : il n'avait pas réuni le nombre requis de signatures d'électeurs dans l'un des 15 gouvernorats. Omar Soliman devait décéder brutalement quelques mois plus tard, en juillet 2012, alors qu'il était aux États-Unis pour des examens médicaux.

14. La loi d'organisation de la Haute Cour constitutionnelle de 1979 (article 29 a) prévoit qu'elle peut être saisie par tout tribunal ou « organe doté d'une compétence juridictionnelle ». Dans sa décision du 14 juin, la Haute Cour constitutionnelle, après avoir examiné la composition et le mandat de la Commission des élections présidentielles, jugea qu'elle répondait aux critères d'un « organe doté d'une compétence juridictionnelle » et avait donc capacité pour la saisir.

15. La Commission des élections présidentielles étant présidée par le président de la Haute Cour constitutionnelle, ce dernier se récusa pour l'examen de la constitutionnalité de la loi sur l'exercice des droits politiques et ne siégea pas. 16. Haute Cour constitutionnelle, 21 juin 1986.

17. Haute Cour constitutionnelle, 4 avril 1987.

18. New York Times, 3 juillet 2012, “Judge Helped Egypt's Military to Cement Power", (http://www.nytimes.com/2012/07/04/world/middleeast/judgehelped-egypts-military-to-cement-power.html?_r=2\&pagewanted=all). Propos démentis ensuite par la magistrate mais confirmés par le journal.

19. Le fait que le procureur général soit toujours en poste alors qu'il avait été nommé par le président Moubarak est souvent avancé comme un des facteurs d'explication de certaines sentences plutôt légères prononcées par les tribunaux à l'égard des policiers accusés d'avoir tiré sur des manifestants, ou de procès contre les anciens hauts dirigeants du régime de Moubarak : les dossiers d'instruction étant incomplets et souvent dépourvus d'éléments concrets de preuve, les juges ne peuvent que relaxer les accusés, sous peine de ne pas rendre justice.

20. La loi d'organisation de la Haute Cour constitutionnelle ne fixe pas le nombre de membres de la Cour, se contentant d'affirmer qu'il doit être « suffisant ».

21. Louis Favoreu "Crise du juge et contentieux constitutionnel en droit français », in J. Lenoble (éd.), La crise du juge, Paris-Bruxelles, LGDJBruylant, coll. "La pensée juridique moderne », 1996, p. 84.

22. Marc Verdussen « La justice constitutionnelle en Europe centrale. Essai de synthèse ", in Marc Verdussen (dir.), La justice constitutionnelle en Europe centrale, Bruylant, Bruxelles, 1997, p. 250. 